\title{
The time-varying shortest path problem with fuzzy transit costs and speedup
}

\author{
Hassan Rezapour \\ Department of Mathematics, \\ Faculty of Basic Sciences, \\ University of Qom, Iran \\ email: Hassan.Rezapour@gmail.com
}

\author{
Gholamhassan Shirdel \\ Department of Mathematics, \\ Faculty of Basic Sciences, \\ University of Qom, Iran \\ email: Shirdel81math@gmail.com
}

\begin{abstract}
In this paper, we focus on the time-varying shortest path problem, where the transit costs are fuzzy numbers. Moreover, we consider this problem in which the transit time can be shortened at a fuzzy speedup cost. Speedup may also be a better decision to find the shortest path from a source vertex to a specified vertex.
\end{abstract}

\section{Introduction}

Time-varying shortest path problem may arise in the applications of mathematics such as transportation, telecommunication and computer networks. The problem is to find the shortest path from a source vertex to a target vertex, so that the total costs of path is minimized subject to the total times of path is at most $\mathrm{T}$, where $\mathrm{T}$ is the time horizon and a given positive integer. The shortest path problem with fuzzy numbers has been studied by Kelvin [7], where a new model based on fuzzy number was presented. Lin and Chern [10] and Li and Gen [9] surveyed this subject, separately. Gent et al. in [3] solved the shortest path problem by genetic algorithm. Shirdel and Rezapour in [15] studied a k-objective time-varying shortest path problem, which cannot

2010 Mathematics Subject Classification: 90C35, 90B10

Key words and phrases: time-varying optimization, shortest path, fuzzy numbers, speedup 
be combined into a single overall objective. Okada and Gen [13] concentrated on the problem with interval numbers. Then, Okada and Soper maintained their work on the shortest path in network with fuzzy number in [14]. We encourage the reader to consult $[2,4,6,8,11,12]$ for historical background, computational techniques and mathematical properties of the fuzzy shortest path problem. In this paper, we consider time-varying shortest path, where transit costs are triangular fuzzy numbers. Moreover, we assume that the transit times and the transit costs are dependent on discrete time $T$, where $T$ is the time horizon. The preliminary and definitions are given in Section 2. The problem is discussed in Section 3 and two theorems are proved for solving of problem. An algorithm is presented in Section 4 for the mentioned problem.

\section{Preliminary}

Consider a time-varying network $G(V, A, b, c)$, where $V$ and $A$ are the set of vertices and the set of arcs, respectively, with $|\mathrm{V}|=\mathrm{n},|\mathrm{A}|=\mathrm{m}$. The transit time $b(i, j, t)$ and the fuzzy transit cost $\tilde{c}(i, j, t)$ are associated with each arc $(i, j)$, respectively, such that $t$ is the departure time of a vertex $i$. Moreover, $\tilde{c}(i, j, t)$ is assumed to be triangular fuzzy number. The transit time $b(i, j, t)$ and the fuzzy transit cost $\tilde{c}(i, j, t)$ are the functions of discrete time $t=0,1, \ldots, T$, where $T$ is a given positive integer. The waiting time at vertex $i$ from $t$ to $t+1$ is shown by $w(i)$ and the associated fuzzy waiting cost is presented by $\tilde{\mathfrak{c}}(i, t)$.

Definition 1 [5] The membership function $\mu_{A}(x): X \rightarrow[0,1]$ allocates a value between 0 or 1 to each member in $\mathrm{X}$, where $\mathrm{X}$ is a universal set and $\mathrm{A} \subseteq \mathrm{X}$. The assigned values point out the membership grade of the element in the set $A$, and moreover the set $\left\{\left(x, \mu_{A}(x)\right): x \in X\right\}$ is named fuzzy set.

Definition 2 [5] A fuzzy number $\tilde{A}=(\alpha, \beta, \gamma)$ is called to be a triangular fuzzy number, when it has the following membership function:

$$
\mu_{\tilde{A}}(x)=\left\{\begin{array}{cc}
\frac{x-\alpha}{\beta-\alpha} & \alpha \leq x \leq \beta \\
1 & x=\beta \\
\frac{\gamma-x}{\gamma-\beta} & \beta \leq x \leq \gamma \\
0 & \text { otherwise }
\end{array}\right.
$$


where, $\alpha \in \mathrm{R}, \beta \in \mathrm{R}$ and $\gamma \in \mathrm{R}$.

Definition 3 [6] Let $\tilde{A}=\left(\alpha_{1}, \beta_{1}, \gamma_{1}\right)$ be a triangular fuzzy number, then its ranking function $\tilde{A}$ is a function $\mathfrak{R}: \mathfrak{R}(\tilde{\mathrm{A}}) \rightarrow \mathbb{R}$, where $\mathfrak{R}(\tilde{\mathrm{A}})$ is the set of all fuzzy numbers. For a triangular fuzzy number $\tilde{A}=\left(\alpha_{1}, \beta_{1}, \gamma_{1}\right)$, the ranking function $\mathfrak{R}$ is calculated by $\mathfrak{R}(\tilde{A})=\frac{1}{4}\left(\alpha_{1}+2 \beta_{1}+\gamma_{1}\right)$.

Definition 4 [6] Assume $\tilde{\mathrm{A}}=\left(\alpha_{1}, \beta_{1}, \gamma_{1}\right)$ and $\tilde{\mathrm{B}}=\left(\alpha_{2}, \beta_{2}, \gamma_{2}\right)$ are two triangular fuzzy numbers, then:

- $\tilde{\mathrm{A}} \oplus \tilde{\mathrm{B}}=\left(\alpha_{1}+\alpha_{2}, \beta_{1}+\beta_{2}, \gamma_{1}+\gamma_{2}\right)$,

- $\tilde{\mathrm{A}}>\tilde{\mathrm{B}}$ if and only if $\mathfrak{R}(\tilde{\mathrm{A}})>\mathfrak{R}(\tilde{\mathrm{B}})$,

- $\tilde{\mathrm{A}}=\tilde{\mathrm{B}}$ if and only if $\mathfrak{R}(\tilde{\mathrm{A}})=\mathfrak{R}(\tilde{\mathrm{B}})$,

- $\tilde{\mathrm{A}}<\tilde{\mathrm{B}}$ if and only if $\mathfrak{R}(\tilde{\mathrm{A}})<\mathfrak{R}(\tilde{\mathrm{B}})$,

- A triangular fuzzy number $\tilde{\mathrm{A}}_{\mathrm{k}}$ is the maximum triangular fuzzy numbers $\tilde{A}_{i}$ such that $\mathfrak{R}\left(\tilde{A}_{k} \geq\right) \mathfrak{R}\left(\tilde{A}_{i}\right)$ for all $1 \leq i \leq n$,

- Minimum triangular fuzzy numbers $\tilde{A}_{i}$ is similarly defined,

- Moreover, let $\tilde{0}=\tilde{A} \Leftrightarrow \alpha_{1}=0, \beta_{1}=0, \gamma_{1}=0$ and $\tilde{A}=\tilde{\infty} \Leftrightarrow \mathfrak{R}(\tilde{A})=\tilde{\infty}$.

Definition 5 [1] Suppose a time-varying path from $i_{1}$ to $i_{k}$ is specified by $\mathrm{P}\left(i_{1}-i_{2}-\cdots-i_{k}\right)$. Consider $\alpha\left(i_{r}\right)$ be the arrival time of a vertex $i_{r}$ on $\mathrm{P}$ such that $\alpha\left(i_{1}\right)=t_{1} \geqslant 0$ and we have:

$$
\alpha\left(i_{r}\right)=\alpha\left(i_{r-1}\right)+w\left(i_{r-1}\right)+b\left(i_{r-1}, i_{r}, \tau\left(i_{r-1}\right)\right) \quad \text { for } 2 \leq r \leq k
$$

where, $\tau\left(i_{r-1}\right)$ is the departure time of a vertex $i_{r-1}$ for for $2 \leq r \leq k$ on $\mathrm{P}$ and we have:

$$
\tau\left(i_{r-1}\right)=\alpha\left(i_{r-1}\right)+w\left(i_{r-1}\right) \quad \text { for } 2 \leq r \leq k .
$$

Moreover, let $\alpha(s)=0$ for the source vertex $\mathrm{s}$.

Definition 6 [1] Let $\mathrm{P}\left(\mathfrak{i}_{1}=\mathrm{s}-\mathfrak{i}_{2}-\cdots-\mathfrak{i}_{\mathrm{k}}\right)$ be a time-varying path from $\mathrm{s}$ to $i_{k}$, then the time of time-varying path $\mathrm{P}$ is determined by $\alpha\left(i_{k}\right)+w\left(i_{k}\right)$.

Definition 7 The fuzzy cost of the time-varying path $\mathrm{P}\left(\mathfrak{i}_{1}-\mathfrak{i}_{2}-\cdots-\mathfrak{i}_{\mathrm{k}}\right)$ is defined as follow:

$$
\tilde{\zeta}(P)=\tilde{\zeta}\left(i_{k}\right)=\tilde{\zeta}\left(i_{k-1}\right)+\tilde{c}\left(i_{k-1}, i_{k}, \tau\left(i_{k-1}\right)\right)+\sum_{t^{\prime}=0}^{w\left(i_{k}\right)-1} \tilde{c}\left(i_{k}, t^{\prime}+\alpha\left(i_{k}\right)\right)
$$

Moreover, the path $\mathrm{P}$ is the shortest path within time $\mathrm{t}$ if for each path $\mathrm{P}^{\prime}$ within time $\mathrm{t}$, we have: $\zeta(\mathrm{P}) \leq \zeta\left(\mathrm{P}^{\prime}\right)$. 


\section{The fuzzy shortest path problem with speed up}

Consider that the transit time $b(i, j, t)$ can be reduced at a fuzzy speedup cost $\tilde{c_{\gamma}}(i, j, t)$ i.e. an arc $(i, j)$ is traversed in shorter time and $b(i, j, t)$ is rebated by paying the speedup cost $\tilde{c_{\gamma}}(i, j, t)$. Speedup on one or several arcs may be leaded to a better solution; especially it may be necessary when the deadline T is tight. Let $\gamma(i, j, t)$ be the amount of time reduced from the transit time $b(i, j, t)$ with fuzzy speedup cost $\tilde{c_{\gamma}}(i, j, t)$, such that $b(i, j, t)-\gamma(i, j, t)>0$.

Theorem 1 Define $\mathrm{d}_{\mathcal{A}}^{s}(\mathfrak{j}, \mathrm{t})$ as the fuzzy cost of a time-varying shortest path from $\mathrm{s}$ to $\boldsymbol{j}$ of time exactly $\mathrm{t}$ with speed up. Then $\mathrm{d}_{\mathrm{A}}^{\mathrm{s}}(\mathbf{j}, 0)=\tilde{\infty}$ for all $\boldsymbol{j} \neq \mathrm{s}$, $\mathrm{d}_{\mathrm{A}}^{\mathrm{s}}(\mathrm{s}, 0)=\tilde{0}$ and if $\mathrm{t}>0$ have:

$$
\begin{gathered}
d_{A}^{s}(j, t)=\min \left\{d_{A}^{s}(j, t-1)+\tilde{c}(j, t-\right. \\
\left.1), \min _{(i, j) \in A} \min _{\mathfrak{u}+b(i, j, u)-\gamma(i, j, t)=t}\left\{d_{A}^{s}(i, u)+\tilde{c}(i, j, u)+\tilde{c}_{\gamma}(i, j, u)\right\}\right\}
\end{gathered}
$$

Proof. It is clear that $d_{A}^{s}(j, 0)=\tilde{\infty}$ for all $j \neq s$ and $d_{A}^{s}(s, 0)=\tilde{0}$, since all transit times are positives. The theorem is proved by induction on $t>0$. Consider $t=1$, for $j=s$ the theorem clearly holds. If $j \neq s$, for $(s, j) \in A$ and $b(s, j, 0)=1$, the theorem holds with $d_{A}^{s}(s, 0)+\tilde{c}(s, j, 0)+\tilde{c}_{\gamma}(s, j, 0)$. Assume that the theorem is correct for $t^{\prime}<t$ and $d_{A}^{s}(j, t)$ is finite. If $d_{A}^{s}(j, t)=$ $d_{\mathcal{A}}^{s}(j, t-1)+\tilde{c}(j, t-1)$, by induction, there is a path from $s$ to $j$ within time $t-1$, by waiting at $j$ one unit of time more, the time of path is exactly $t$. If $d_{A}^{s}(j, t)=d_{A}^{s}(i, u)+\tilde{c}(i, j, u)+\tilde{c}_{\gamma}(i, j, u)$, since $b(i, j, t)-\gamma(i, j, t)>0$, then $u<t$, therefore by induction, there is a path from $s$ to $i$ within time $u$ and cost $d_{A}^{s}(i, u)$. We can extend this path to $j$, obtaining a path from $s$ to $j$ within time $u+b(i, j, u)-\gamma(i, j, t)=t$ and cost $d_{A}^{s}(j, t)=d_{A}^{s}(i, u)+\tilde{c}(i, j, u)+\tilde{c}_{\gamma}(i, j, u)$. It is easy to see that $d_{\mathcal{A}}^{s}(j, t)$ is the fuzzy cost of shortest path from $s$ to $j$.

Theorem 2 Define $\mathrm{d}_{A}^{\mathrm{s}^{*}}(\mathrm{j})$ as the cost of a time-varying shortest path form $\mathrm{s}$ to $\mathrm{j}$ of time at most $\mathrm{T}$ with speed-up, then we have:

$$
d_{A}^{s^{*}}(j)=\min _{0 \leq t \leq T} d_{A}^{s}(j, t) .
$$

Proof. The proof is Straightforward.

\section{The algorithm for solving fuzzy shortest path prob- lem with speed up}

The key idea in the below algorithm is to first sort the values of $u+b(i, j, u)-$ $\gamma(i, j, t)=t$ for all $u=0,1,2, \ldots, T$ and all arcs $(i, j) \in A$, before the recursive 
relation as given in theorem 1 is applied to compute $d_{A}^{s}(j, t)$ for all $j \in V$ and $\mathrm{t}=0,1,2, \ldots, \mathrm{T}$.

\section{Algorithm}

\section{Begin}

2. Let $d_{A}^{s}(j, 0)=\tilde{\infty}$ for all $j \neq s, d_{A}^{s}(s, 0)=\tilde{0}$;

3. Sort all values $u+b(i, j, u)-\gamma(i, j, t)=t$ for all $u=0,1,2, \ldots, T$ and all $\operatorname{arcs}(i, j) \in A$;

4. For $\mathrm{t}=0,1,2, \ldots, \mathrm{T}$, do;

For $j \in V$, do;

For each $(i, j) \in A$, and each $u$ and $\gamma$, do;

$$
\begin{gathered}
d_{A}^{s}(j, t)=\min \left\{d_{A}^{s}(j, t-1)+\tilde{c}(j, t-\right. \\
\left.1), \min _{(i, j) \in A} \min _{\mathfrak{u}+b(i, j, t)-\gamma(i, j, t)=t}\left\{d_{A}^{s}(i, u)+\tilde{c}(i, j, u)+\tilde{c}_{\gamma}(i, j, u)\right\}\right\}
\end{gathered}
$$

5. Let $d_{A}^{s^{*}}(j)=\min _{0 \leq t \leq T} d_{A}^{s}(j, t)$;

6. End

Example 1 Consider a given time-varying network $\mathrm{G}$ in Figure 1, where $\mathrm{T}=6$.

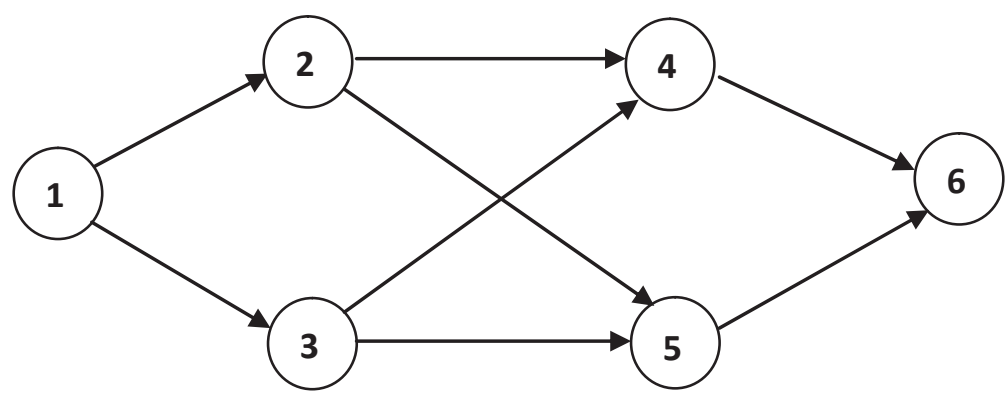

Figure1. A network for example 1

Assume that the waiting at vertices are not allowed, i.e. $\mathfrak{w}(\mathfrak{i})=0$ for all $\mathfrak{i} \in \mathrm{V}$. Furthermore, for arcs $(1,2),(1,3)$ and $(2,4)$ and for each time $\mathrm{t}=0,1, \ldots, 6$ let: 


$$
\mathrm{b}(i, j, t)=3, \tilde{c}(i, j, t)=(2,3,4)
$$

for arcs $(4,6)$ and $(5,6)$ and for each time $\mathrm{t}=0,1, \ldots, 6$ let:

$$
\mathrm{b}(i, j, t)=3, \tilde{c}(i, j, t)=(1,3,5)
$$

Other transit times and fuzzy transit costs are shown in Table 1.

\begin{tabular}{|c|c|c|c|c|c|c|}
\hline Arcs & \multicolumn{2}{|c|}{$(2,5)$} & \multicolumn{2}{|c|}{$(3,4)$} & \multicolumn{2}{|c|}{$(3,5)$} \\
\hline $\mathbf{b}, \tilde{c}$ & b & $\tilde{\boldsymbol{c}}$ & b & $\tilde{\boldsymbol{c}}$ & b & $\tilde{\boldsymbol{c}}$ \\
\hline 0 & 1 & $(1,2,3)$ & 2 & $(2,4,6)$ & 3 & $(1,4,5)$ \\
\hline 1 & 4 & $(1,3,4)$ & 2 & $(2,4,5)$ & 2 & $(1,4,6)$ \\
\hline 2 & 3 & $(1,3,4)$ & 1 & $(3,4,5)$ & 3 & $(2,3,4)$ \\
\hline 3 & 3 & $(2,3,5)$ & 4 & $(3,4,6)$ & 5 & $(2,4,6)$ \\
\hline 4 & 2 & $(1,3,6)$ & 3 & $(1,3,5)$ & 4 & $(3,5,6)$ \\
\hline 5 & 3 & $(1,3,5)$ & 2 & $(1,3,4)$ & 3 & $(4,5,6)$ \\
\hline 6 & 4 & $(1,3,4)$ & 3 & $(1,2,4)$ & 2 & $(2,5,7)$ \\
\hline
\end{tabular}

Table 1. Information for network $G$

There is not any feasible path from source vertex 1 to vertex 6 with $\mathrm{T}=6$, because each path has time more than time horizon $\mathrm{T}=6$. Let $\gamma(\mathrm{i}, \mathrm{j}, \mathrm{t})=1$, corresponding to each $\gamma$, consider that there is a speedup cost $\tilde{\mathbf{c}}_{\gamma}(\mathbf{i}, \mathbf{j}, \mathrm{t})=$ $(2,4,6)$. After applying the described algorithm to find the shortest path between the vertex 1 and the vertex 6, we can obtain a path 1-2-5-6 with fuzzy cost $\mathrm{d}_{\mathrm{A}}^{\mathrm{s}^{*}}(\mathrm{j})=(10,20,31)$.

\section{Conclusion}

In the time-varying shortest path problem, speedup may be a better decision for the solution, although it incurs an extra cost. In particular, speedup may 
become necessary when the deadline $\mathrm{T}$ is tight. In this paper, we have considered one class of the time-varying shortest path, where the transit costs are fuzzy numbers and speedups on all arcs along the path are decision variables. Moreover, we have presented an algorithm for solving the problem.

\section{References}

[1] X. Cai X, D. Sha, C. K. Wong, Time-varying network optimization, Springer Science Press. New York, USA, 2007.

[2] L. Campos, J. L. Verdegay, Linear programming problems and ranking of fuzzy numbers, Fuzzy Sets and Systems, 32 (1989), 1-11.

[3] M. Gent, R. Cheng, D. Wang, Genetic algorithms for solving shortest path problems, Proceedings of the IEEE International Conference on Evolutionary Computation, (1997), 401-406.

[4] X. Ji, K. Iwamura, Z. Shao, New models for shortest path problem with problem with fuzzy arc lengths, Applied Mathematical Modeling, 31 (2007), 259-269.

[5] A. Kaufmann, M. M. Gupta, Fuzzy mathematical models in engineering and management science, Elsevier, Amsterdam, The Netherland, 1988.

[6] A. Kaur, A. Kumar, A new approach for solving fuzzy transportation problems using generalized trapezoidal fuzzy numbers, Applied Soft Computing, 12 (2012), 1201-1213.

[7] C. M. Klein, Fuzzy shortest path, Fuzzy Sets and Systems, 39 (1991), $27-41$.

[8] A. Kumar, K. Manjot, A new algorithm for solving network flow problems with fuzzy arc lengths, Turkish Journal of Fuzzy Systems, 1 (2011), 1-13.

[9] Y. Li, M. Gen, K. Ida, Solving fuzzy shortest path problems by neural networks, Computers and Industrial Engineering, 31 (1996), 861-865.

[10] K. C. Lin, M. S. Chern, The fuzzy shortest path problem and its most vital arcs, Fuzzy Sets and Systems, 58 (1993), 343-353.

[11] I. Mahdavi, R. Nourifar, A. Heidarzade, N. M. Amiri, A dynamic programming approach for finding shortest chains in fuzzy network, Applied Soft Computing, 9 (2009), 503-511. 
[12] S. Moazeni, Fuzzy shortest path problem with finite fuzzy quantities, Applied Mathematics and Computation, 183 (2006), 160-169.

[13] S. Okada, M. Gen, Fuzzy shortest path problems, Computers and Industrial Engineering, 27 (1994), 465-468.

[14] S. Okada, T. Soper, A shortest path problem on a network with fuzzy arc lengths, Fuzzy Sets and Systems, 109 (2000), 129-140.

[15] Gh. Shirdel, H. Rezapour, K-Objective Time-Varying Shortest Path Problem with Zero Waiting Times at Vertices, Trends in Applied Science Research, 5 (2015), 278-285.

Received: May 16, 2016 